\title{
Salmonella typhimurium Strain LT-2 is still Pathogenic for Man
}

\author{
By S. BAUMBERG \\ Department of Genetics, University of Leeds, Leeds 2 \\ AND R. FREEMAN \\ Department of Bacteriology, University of Leeds, Leeds 2 \\ (Accepted for publication 19 November 1970 )
}

During a class experiment in the Department of Genetics, University of Leeds, an undergraduate using an unplugged pipette swallowed about $2 \mathrm{ml}$. of a soft agar suspension containing Salmonella typhimurium strain LT-2 together with its temperate phage P 22 (the count was almost certainly greater than $10^{8}$ bacteria $/ \mathrm{ml}$.). She experienced mild gastritis for the next 3 days, but felt better on the fourth. On the fifth day, her condition worsened, the symptoms including headache, malaise, and shortly afterwards diarrhoea; the following day, these all became more pronounced and vomiting occurred several times during the night. Within the next $24 \mathrm{~h}$. there was considerable improvement and 3 days later she was completely recovered.

A stool specimen was taken ro days after the ingestion, and was cultured in the standard way. A primary culture was negative, but a culture made from a portion of the specimen which had been incubated in Selenite F broth for $48 \mathrm{~h}$. yielded an almost pure growth of an organism with the following characteristics: it gave no fermentation reaction with sucrose or lactose, but produced acid + gas with dulcitol, mannitol and glucose; did not produce indole; and was highly motile. Agglutination tests with specific antisera showed that it possessed somatic and flagellar antigens specific to Salmonella typhimurium. The strain used in the practical class was tested for all the above characters, and behaved in an identical manner. Furthermore, the isolated organism required proline for growth on minimal salts + glucose medium, as did the class strain (proA 107, originally from the late Dr M. Demerec's collection).

Finally, both strains were sent to the Central Enteric Reference Laboratory, Colindale, London. Investigations there confirmed that the two organisms were Salmonella typhimurium and that they belonged to the same phage type (Ib).

In summary, this patient suffered an attack of salmonellosis following accidental ingestion of Salmonella typhimurium strain LT-2. An organism isolated from the faeces was identical to the original strain. We conclude that the case indicates that this strain can still, on occasion, act as a pathogen for man.

Salmonella typhimurium LT-2 is widely used in research and teaching in bacterial and molecular genetics. It is sometimes regarded as being non-pathogenic and is treated accordingly; for instance, a standard textbook (Clowes \& Hayes, 1968) says: 'The micro-organisms employed in the experiments are of non-pathogenic groups such as Escherichia coli, except for Salmonella typhimurium strain LT-2. However, this latter strain has been used for many years in laboratory experiments and it is generally agreed to be harmless. Accordingly, the use of unplugged pipettes for dispensing all cultures, including LT-2 (saving much time in cleaning and preparing pipettes for reuse) 
is now common practice, and is considered a safe procedure.' We should like to make clear that the procedure during which the accidental ingestion occurred (transferring a soft agar lysate by pipette from Petri dish to centrifuge tube) is nowhere recommended by these authors; they advocate the liquid lysate technique for preparing phage $\mathrm{P} 22$ stocks. In the light of this incident, it would appear that the organism, 19 years after the discovery of its capacity to be transduced (Lederberg, Lederberg, Zinder \& Lively, I95I), since when it has presumably been maintained on laboratory culture media, retains some mildly unpleasant potentialities; and that certain additional precautions, such as pipetting with plugged pipettes or with a teat, may be advisable.

We should like to thank the Director, Dr E. S. Anderson, and members of the technical staff of the Central Enteric Reference Laboratory, Colindale, for their help, and Professor W. Hayes for his comments.

\section{REFERENCES}

Clowes, R. C. \& HAYES, W. (I968). In Experiments in Microbial Genetics, p. I I. Edited by R. C. Clowes \& W. Hayes. Oxford and Edinburgh: Blackwell Scientific Publications.

LeDERBERG, J., LEDERBERG, E. M., ZINDER, N. D. \& LIVELY, E. R. (1951). Recombination analysis of bacterial heredity. Cold Spring Harbor Symposia on Quantitative Biology 16, 4I3-44I. 